\title{
Citrobacter freundii Complex
}

National Cancer Institute

\section{Source}

National Cancer Institute. Citrobacter freundii Complex. NCI Thesaurus. Code C86266.

A non taxonomic grouping of bacteria assigned to and including eight related species from the Citrobacter genus. 